\title{
How a Minimum Time Step and Formation of Initial Causal Structure in Space-Time Is Linked to an Enormous Initial Cosmological Constant
}

\author{
Andrew Walcott Beckwith \\ Physics Department, College of Physics, Chongqing University, Chongqing, China \\ Email: Rwill9955b@gmail.com, abeckwith@uh.edu
}

How to cite this paper: Beckwith, A.W. (2018) How a Minimum Time Step and Formation of Initial Causal Structure in Space-Time Is Linked to an Enormous Initial Cosmological Constant. Journal of High Energy Physics, Gravitation and Cosmology, 4, 541-548.

https://doi.org/10.4236/jhepgc.2018.43032

Received: May 7, 2018

Accepted: July 28, 2018

Published: July 31, 2018

Copyright $\odot 2018$ by author and Scientific Research Publishing Inc. This work is licensed under the Creative Commons Attribution International License (CC BY 4.0).

http://creativecommons.org/licenses/by/4.0/

\begin{abstract}
We use a root finder procedure to obtain $\Delta t$ and an inflaton value due to use of a scale factor $a \sim a_{\text {min }} t^{\gamma}$ if we furthermore use $\delta g_{t t} \sim a_{\text {min }}^{2} \cdot \phi_{\text {initial }}$ as the variation of the time component of the metric tensor $g_{t t}$ in Pre-Planckian Space-time up to the Planckian space-time initial values. In doing so, we obtain, due to the very restricted values for $\Delta t$ which are of the order of less than Planck time, results leading to an enormous value for the initial Cosmological constant.
\end{abstract}

\section{Keywords}

Inflaton Physics, Causal Structure, Non-Linear Electrodynamics

\section{Framing the Initial Inquiry}

Volovik [1] derives in page 24 of his manuscript a description of a total vacuum energy via an integral over three-dimensional space

$$
E_{\text {Vac }}(N)=\int \mathrm{d}^{3} r \cdot \varepsilon(n)
$$

The integrand to be considered is, using a potential defined by $U=\frac{c^{2} m}{n}$ as given by Volovik for weakly interacting Bose gas particles, as well as

$$
\varepsilon(n)=\frac{1}{2} U \cdot n^{2}+\frac{8}{15 \pi^{2} \hbar^{3}} m^{3 / 2} U^{5 / 2} n^{5 / 2}=\frac{1}{2} \cdot c^{2} \cdot\left[n \cdot m+\frac{4}{15} \cdot\left(\frac{m^{5}}{\hbar^{2} \cdot \sqrt{c}}\right) \cdot \frac{1}{n^{2}}\right](2)
$$

For the sake of argument, $m$, as given above will be called the mass of a graviton, $n$ a numerical count of gravitons in a small region of space, and afterwards, adaptations as to what this expression means in terms of entropy generation which will be subsequently raised. A simple graph of the $2^{\text {nd }}$ term of Equation (2) 
with comparatively large $m$ and with $\hbar=c=1$ has the following qualitative behavior, namely for

$$
E 1=\left[c^{2} / 2\right] \cdot\left[\frac{4}{15} \cdot\left(\frac{m^{5}}{\hbar^{2} \cdot \sqrt{c}}\right) \cdot \frac{1}{n^{2}}\right]
$$

$E 1 \neq 0$ when $n$ is very small, and $E 1=0$ as $n \rightarrow 10^{10}$ at the onset of inflation. This will tie directly with a linkage between energy and entropy, as seen in the construction, looking at what Kolb [2] put in, i.e.

$$
\rho=\rho_{\text {radiation }}=(3 / 4) \cdot\left[\frac{45}{2 \pi^{2} g_{*}}\right]^{1 / 3} \cdot S^{4 / 3} \cdot r^{-4}
$$

Here, the idea would be, to make the following equivalence. Namely look at,

$$
\left[\left[\frac{\Lambda_{\mathrm{Max}} r^{4}}{8 \pi G}\right] \cdot(4 / 3) \cdot\left[\frac{2 \pi^{2} g_{*}}{45}\right]^{1 / 3}\right]^{3 / 4} \sim S_{\text {initial }}
$$

We furthermore make the assumption of a minimum radius of

$$
R_{\text {initial }} \sim \frac{1}{\#} \ell_{N g}<l_{\text {Planck }}
$$

This Equation (6) will be put as the minimum value of $r$, in Equation (5), where we have in this situation [3] [4]

$$
\text { \#bits } \sim\left[\frac{E}{\hbar} \cdot \frac{l}{c}\right]^{3 / 4} \approx\left[\frac{M c^{2}}{\hbar} \cdot \frac{l}{c}\right]^{3 / 4}
$$

And if $M$ is the total space-time energy mass, for initial condition and $E 1$ is the main fluctuation in energy we have to consider, if $\Delta E \sim E 1$, as well as [3] [4]

$$
S_{\text {initial }} \sim n_{\text {graviton }} \sim \text { initial graviton count }
$$

Then what can be said about the inter relationship of graviton counts, and the onset of Causal structure?

\section{Examination of the Minimum Time Step, in Pre-Planckian Space-Time as a Root of a Polynomial Equation}

We initiate our work, citing [5] to the effect that we have a polynomial equation for the formation of a root finding procedure for $\Delta t$, namely if

$$
\begin{aligned}
& \Delta t \cdot\left|\left(\sqrt{\frac{8 \pi G V_{0}}{\gamma \cdot(3 \gamma-1)}} \cdot \Delta t-1\right)-\frac{\left(\sqrt{\frac{8 \pi G V_{0}}{\gamma \cdot(3 \gamma-1)}} \cdot \Delta t-1\right)^{2}}{2}+\frac{\left(\sqrt{\frac{8 \pi G V_{0}}{\gamma \cdot(3 \gamma-1)}} \cdot \Delta t-1\right)^{3}}{3}-\cdots\right| \\
& \approx\left(\sqrt{\frac{\gamma}{\pi G}}\right)^{-1} \frac{48 \pi \hbar}{a_{\text {min }}^{2} \cdot \Lambda}
\end{aligned}
$$

From here, we then cited, in [5], using [6] a criteria as to formation of entropy, i.e. if $\Lambda$ is an invariant cosmological "constant" and if Equation (10) holds, 
we can use the existence of nonzero initial entropy as the formation point of an arrow of time.

$$
\left.S_{\Lambda}\right|_{\text {Arrow-of-time }}=\pi \cdot\left(\frac{\left.R_{c}\right|_{\text {initial }} \sim c \cdot \Delta t}{l_{\text {Planck }}}\right)^{2} \neq 0
$$

This leads to the following, namely in [5] we make our treatment of the existence of causal structure, as given by writing its emergence as contingent upon having

$$
\left(\frac{\left.R_{c}\right|_{\text {initial }} \sim c \cdot \Delta t}{l_{\text {Planck }}}\right) \sim \vartheta(1)
$$

The rest of this article will be contingent upon making the following assumptions. FTR

$$
\begin{aligned}
& \Delta E \sim E 1 \\
& S_{\text {initial }} \sim n_{\text {graviton }} \sim \text { initial graviton count } \\
& {\left[\left[\frac{\Lambda_{\mathrm{Max}} r^{4}}{8 \pi G}\right] \cdot(4 / 3) \cdot\left[\frac{2 \pi^{2} g_{*}}{45}\right]^{1 / 3}\right]^{3 / 4} \sim S_{\text {initial }}} \\
& \Leftrightarrow\left[\left[\frac{\Lambda_{\mathrm{Max}} r^{4}}{8 \pi G}\right] \cdot(4 / 3) \cdot\left[\frac{2 \pi^{2} g_{*}}{45}\right]^{1 / 3}\right]^{3 / 4} \sim n_{\text {graviton }} \\
& \Delta E \sim E 1 \sim V_{0} \\
& r \sim R_{\text {initial }} \sim \frac{1}{\#} \ell_{N g}<l_{\text {Planck }}
\end{aligned}
$$

In short, our view is that the formation of a minimum time step, if it satisfies Equation (11) is a necessary and sufficient condition for the formation of an arrow of time, at the start of cosmological evolution we have a necessary and sufficient condition for the initiation of an arrow of time. With causal structure, along the lines of Dowker, as in [7] and given more detail by Equation (12) above as inputs into Equation (10) and Equation (11) i.e. Planck length is set equal to 1 and.

$$
\begin{aligned}
& \left.\underset{\text { Volume }}{\frac{\Delta E \Delta t}{\text { (Pre-Planckian }) \rightarrow(\text { Planckian })}} \Delta \Delta E \Delta t \sim \hbar\right|_{\text {Planckian }}
\end{aligned}
$$

i.e. the regime of where we have the initiation of causal structure, if allowed would be contingent upon the behavior of [5] [8] [9]

$$
\begin{aligned}
& g_{t t} \sim \delta g_{t t} \approx a_{\min }^{2} \phi_{\text {initial }} \ll 1 \\
& \Leftrightarrow g_{t t} \approx a_{\text {min }}^{2} \phi_{\text {Planck }} \sim 1 \\
& \left.\Leftrightarrow\left(\frac{\left.R_{c}\right|_{\text {initial }} \sim c \cdot \Delta t}{l_{\text {Planck }}}\right) \sim \vartheta(1)\right|_{\text {Planck }}
\end{aligned}
$$

i.e. the right hand side of Equation (14) is the square of the scale factor, which we assume is $\sim 10^{\wedge}-110$, due to [4] [10], and an inflaton given by [4] [11] 
So, the question well will be leading up to is what does Equation (9), Equation (12), and Equation (13), tell us about graviton production, and the causal foundation condition stated at Equation (14)?

\section{Conclusion, So What Is the Root of Our Approximation for a Time Step?}

Here our derivation result which satisfies Equation (14) is contingent upon initial $\left.R_{c}\right|_{\text {initial }} \sim c \cdot \Delta t$ as an initial event horizon. So, our bubble of space-time is of the order of magnitude of approximately one Planck Length,

$$
\begin{aligned}
& \left.\frac{\Delta E}{\text { Volume }}\right|_{\text {Pre-Planck }} \\
& \left.\sim\left[\hbar /\left(\Delta t \cdot\left(\text { Volume } \equiv\left(\frac{l_{N g}}{\#}\right)^{3}\right) \cdot\left(\delta g_{t t} \sim a_{\text {min }}^{2} \cdot \phi_{\text {initial }}\right)\right)\right]\right|_{\text {Pre-Planckian }} \\
& \sim \frac{c^{2} \cdot m_{\text {graviton }}^{2}}{30 \hbar^{2} \sqrt{c}} \cdot\left(\text { Volume } \equiv\left(\frac{l_{N g}}{\#}\right)^{3}\right)^{-1} \cdot\left(\frac{1}{n_{\text {boson }}=n_{\text {graviton }}}\right)^{2} \\
& \underset{(\text { Pre-Planckian }) \rightarrow(\text { Planckian })}{\longrightarrow} \\
& \left.\left.\left.\Delta E\right|_{\text {Planckian }} \sim \frac{\hbar}{\Delta t}\right|_{\text {Planckian }} \cdot\left(\text { Volume } \equiv\left(l_{\text {Planck }}\right)^{3}\right)^{-1} \sim \frac{\hbar}{\Delta t}\right|_{\text {Planckian }}
\end{aligned}
$$

A convenient normalization would be to have

$$
\begin{aligned}
& r \sim R_{\text {initial }} \sim \frac{1}{\#} \ell_{N g}<l_{\text {Planck }} \\
& \& \ell_{N g} \sim l_{\text {Planck }} \equiv 1 \\
& \& r \sim R_{\text {initial }} \sim \frac{1}{\#}
\end{aligned}
$$

If so then, Equation (14) would read as a causal formation transformation we would give as

$$
\begin{aligned}
& r \sim R_{\text {initial }} \sim \frac{1}{\#} \ell_{N g}<l_{\text {Planck }} \& \ell_{N g} \sim l_{\text {Planck }} \equiv 1 \\
& \& r \sim R_{\text {initial }} \sim \frac{1}{\#} \& \hbar \equiv c \equiv 1 \\
& \left.\frac{\Delta E}{\text { Volume }}\right|_{\text {Pre-Planck }} \sim\left[1 /\left.\left(\Delta t \cdot\left(\text { Volume } \equiv\left(\frac{1}{\#}\right)^{3}\right) \cdot\left(\delta g_{t t} \sim a_{\text {min }}^{2} \cdot \phi_{\text {initial }}\right)\right)\right|_{\text {Pre-Planckian }}\right. \\
& \sim \frac{m_{\text {graviton }}^{2}}{30} \cdot\left(\text { Volume } \equiv\left(\frac{1}{\#}\right)^{3}\right)^{-1} \cdot\left(\frac{1}{n_{\text {boson }}=n_{\text {graviton }}}\right)^{2} \\
& \left.\left.\underset{\text { (Pre-Planckian) } \rightarrow(\text { Planckian) }}{\longrightarrow} \Delta E\right|_{\text {Planckian }} \sim \frac{1}{\Delta t}\right|_{\text {Planckian }}
\end{aligned}
$$

And then we would have the following equation if we make the following fur- 
ther normalization, as to Planck Mass, and Graviton mass, namely Planck Mass $\sim 2.17645 \mathrm{e}-5$ grams, whereas $M$ (graviton) 2.1e-62 grams. i.e. If Planck Mass $=1$ in normalization, then $M$ (graviton) $\sim 10^{\wedge}-57$

$$
\begin{aligned}
& \left.\frac{\Delta E}{\text { Volume }}\right|_{\text {Pre-Planck }} \\
& \left.\sim\left[1 /\left(\Delta t \cdot\left(\text { Volume } \equiv\left(\frac{1}{\#}\right)^{3}\right) \cdot\left(\delta g_{t t} \sim a_{\text {min }}^{2} \cdot \phi_{\text {initial }}\right)\right)\right]\right|_{\text {Pre-Planckian }} \\
& \sim \frac{10^{-114}}{30} \cdot\left(\frac{\#^{3}}{\left(n_{\text {boson }}=n_{\text {graviton }}\right)^{2}}\right) \sim \frac{10^{-114}}{30} \\
& \left.\left.\frac{(\text { Pre-Planckian }) \rightarrow(\text { Planckian })}{\longrightarrow} \Delta E\right|_{\text {Planckian }} \sim \frac{1}{\Delta t}\right|_{\text {Planckian }} \sim \mathrm{O}(1)
\end{aligned}
$$

i.e. we would roughly have

$$
\left.\frac{10^{-114}}{30} \underset{\text { (Pre-Planckian) } \rightarrow \text { (Planckian) }}{\longrightarrow} \frac{1}{\Delta t}\right|_{\text {Planckian }} \sim \mathrm{O}(1)
$$

This outlines the enormity of the change from Pre Planckian to Planckian physics. If this is true, it indicates the enormity of the Pre Planckian to Planckian transformation. If we assume that $a_{\min }^{2}$ remains invariant, it means that the contribution of the inflaton becomes almost infinitely larger, i.e. $a_{\min }^{2} \sim$ $10^{\wedge}-110$ in size.

So, if we have

$$
\left.\Delta E\right|_{\text {Pre-Planckian }} \sim \frac{10^{-114}}{30} \cdot\left(\frac{\#^{3}}{\left(n_{\text {boson }}=n_{\text {graviton }}\right)^{2}}\right) \sim \frac{10^{-114}}{30} \sim V_{0}
$$

and if $\left.\Delta E\right|_{\text {Pre-Planckian }} \sim \frac{10^{-114}}{30}$, so that we have

$$
\begin{aligned}
& \Delta t \cdot\left(\sqrt{\frac{8 \pi \cdot\left(\left.\Delta E\right|_{\text {Pre-Planckian }}\right)}{\gamma \cdot(3 \gamma-1)}} \cdot \Delta t-1\right)-\frac{\left(\sqrt{\frac{8 \pi\left(\left.\Delta E\right|_{\text {Pre-Planckian }}\right)_{0}}{\gamma \cdot(3 \gamma-1)}} \cdot \Delta t-1\right)^{2}}{2} \\
& +\frac{\left(\sqrt{\frac{8 \pi \cdot\left(\left.\Delta E\right|_{\text {Pre-Planckian }}\right)}{\gamma \cdot(3 \gamma-1)}} \cdot \Delta t-1\right)^{3}}{3}-\cdots \cdot \approx\left(\sqrt{\frac{\gamma}{\pi}}\right)^{-1} \frac{48 \pi}{a_{\min }^{2} \cdot \Lambda}
\end{aligned}
$$

As 


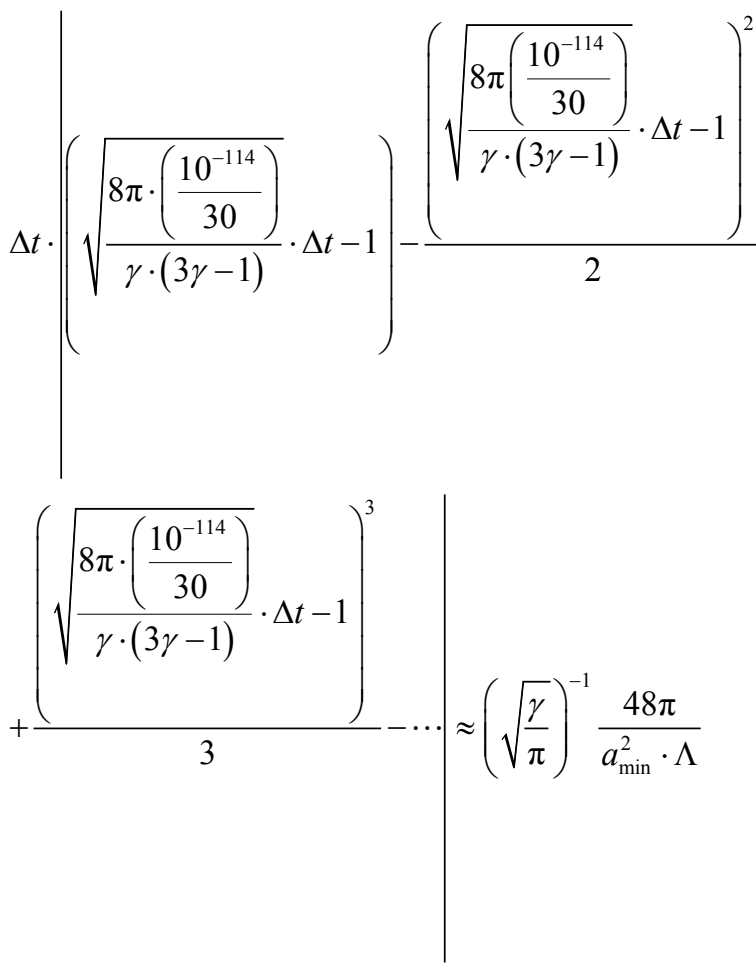

Or more approximately as

$$
\begin{aligned}
& \Delta t \cdot\left|\left(\sqrt{\frac{10^{-114}}{\gamma \cdot(3 \gamma-1)}} \cdot \Delta t-1\right)-\frac{\left(\sqrt{\frac{10^{-114}}{\gamma \cdot(3 \gamma-1)}} \cdot \Delta t-1\right)^{2}}{2}+\frac{\left(\sqrt{\left.\frac{10^{-114}}{\gamma \cdot(3 \gamma-1)} \cdot \Delta t-1\right)^{3}}\right.}{3}-\cdots\right| \\
& \approx\left(\sqrt{\frac{\gamma}{\pi}}\right)^{-1} \frac{48 \pi}{a_{\min }^{2} \cdot \Lambda}
\end{aligned}
$$

Now, set $\Lambda_{\text {initial }}=\Lambda$

$$
\begin{aligned}
& \Delta t \cdot \mid\left(\sqrt{\left.\frac{10^{-114}}{\gamma \cdot(3 \gamma-1)} \cdot \Delta t-1\right)-\frac{\left(\sqrt{\frac{10^{-114}}{\gamma \cdot(3 \gamma-1)}} \cdot \Delta t-1\right)^{2}}{2}+\frac{\left(\sqrt{\left.\frac{10^{-114}}{\gamma \cdot(3 \gamma-1)} \cdot \Delta t-1\right)^{3}}\right.}{3}-\cdots \mid}\right. \\
& \approx\left(\sqrt{\frac{\gamma}{\pi}}\right)^{-1} \frac{48 \pi}{\left(a_{\text {min }}^{2} \sim 10^{-110}\right) \cdot \Lambda_{\text {initial }}} \\
& \approx \Delta t \leq t_{\text {Planck }} \sim 1 \\
& \Leftrightarrow \frac{48 \pi}{\left(a_{\text {min }}^{2} \sim 10^{-110}\right) \cdot \Lambda_{\text {initial }}} \sim \Delta t \leq t_{\text {Planck }} \sim 1 \\
& \Leftrightarrow \Lambda_{\text {initial }} \geq 10^{112}
\end{aligned}
$$


This is on the order of the Cosmological constant, as computed by [12] and Peskins, in [13] so that the Pre Planckian Cosmological constant would have an enormous value on par with the Quantum field theory estimate of the Plancks constant, in Pre Planckian space-time [12] [13].

This so happens to be consistent with Equation (5) of our document. It also has some similarities with the ideas given in [14].

Finally this should be seen in the light of [15] [16] [17] which establish a non-linear electrodynamic treatment of initial singularities, which the author views credible, as an alternative to [18] and the Penrose Singularity theorem.

\section{Acknowledgements}

This work is supported in part by National Nature Science Foundation of China grant No. 11375279.

\section{References}

[1] Volovik, G. (2003) The Universe in a Helium Droplet. International Series of Monographs on Physics, Volume 117. Oxford Press, Oxford.

[2] Kolb, E. and Turner, S. (1994) The Early Universe. Westview Press, Chicago.

[3] Ng, Y.J. (2007) Holographic Foam, Dark Energy and Infinite Statistics. Physics Letters B, 657, 10-14. https://doi.org/10.1016/j.physletb.2007.09.052

[4] Ng, Y.J. (2008) Spacetime Foam: From Entropy and Holography to Infinite Statistics and Nonlocality. Entropy, 10, 441-461. https://doi.org/10.3390/e10040441

[5] Beckwith, A. (2017) How a Minimum Time Step Based in Pre Planckian Space-Time If Friedman Equation H Set Equal to Zero Leads to the Arrow of Time. http://vixra.org/abs/1702.0290

[6] Keifer, C. (2012) Can the Arrow of Time Be Understood from Quantum Cosmology? In: Mersini-Houghton, L. and Vaas, R., Eds., The Arrows of Time, A Debate in Cosmology, Fundamental Theories in Physics, Volume 172, Springer Verlag, Heidelberg, Federal Republic of Germany, 191-203.

[7] Dowker, F. (2005) Causal Sets and the Deep Structure of Spacetime. arXiv:gr-qc/0508109

[8] Beckwith, A. (2016) Gedanken Experiment for Refining the Unruh Metric Tensor Uncertainty Principle via Schwarzschild Geometry and Planckian Space-Time with Initial Nonzero Entropy and Applying the Riemannian-Penrose Inequality and Initial Kinetic Energy for a Lower Bound to Graviton Mass (Massive Gravity). Journal of High Energy Physics, Gravitation and Cosmology, 2, 106-124.

https://doi.org/10.4236/jhepgc.2016.21012

[9] Giovannini, M. (2008) A Primer on the Physics of the Cosmic Microwave Background. World Press Scientific, Hackensack, New Jersey.

https://doi.org/10.1142/6730

[10] Padmanabhan, T. (2005) Understanding Our Universe; Current Status, and Open Issues. In: 100 Years of Relativity, Space-Time, Structure: Einstein and Beyond, World Scientific, P.T.E. LTD, Singapore, 175-204. https://doi.org/10.1142/9789812700988_0007

[11] Camara, C.S., de Garcia Maia, M.R., Carvalho, J.C. and Lima, J.A.S. (2004) Nonsingular FRW Cosmology and Non Linear Dynamics. 
[12] Padmanabhan, T. (2016) Quantum Field Theory, the Why, What and How. Graduate Texts in Physics, Springer Verlag, New York. https://doi.org/10.1007/978-3-319-28173-5

[13] Peskins, M. and Schroeder, D. (1995) An Introduction to Quantum Field Theory. The Advanced Book Program, Perseus Books, Cambridge.

[14] Rovelli, C. and Vidotto, F. (2015) Covariant Loop Quantum Gravity. Cambridge University Press, Cambridge.

[15] Corda, C. and Mosquera Cuesta, H.J. (2011) Inflation from $R^{2}$ Gravity: A New Approach Using Nonlinear Electrodynamics. Astroparticle Physics, 34, 587-590.

[16] De Lorenci, V.A., Klippert, R., Novello, M. and Salim, J.M. (2002) Nonlinear Electrodynamics and FRW Cosmology. Physical Review D, 65, Article ID: 063501.

[17] Corda, C. and Mosquera Cuesta, H.J. (2010) Removing Black-Hole Singularities with Nonlinear Electrodynamics. Modern Physics Letters A, 25, 2423-2429. https://arxiv.org/abs/0905.3298 https://doi.org/10.1142/S0217732310033633

[18] Penrose, R. (1965) Gravitational Collapse and Space-Time Singularities. Physical Review Letters, 14, 57. 